\title{
BETTI NUMBERS OF SEMIALGEBRAIC AND SUB-PFAFFIAN SETS
}

\author{
A. GABRIELOV, N. VOROBJOV AND T. ZELL
}

\begin{abstract}
Let $X$ be a subset in $[-1,1]^{n_{0}} \subset \mathbb{R}^{n_{0}}$ defined by a formula

$$
X=\left\{\mathbf{x}_{0} \mid Q_{1} \mathbf{x}_{1} Q_{2} \mathbf{x}_{2} \ldots Q_{\nu} \mathbf{x}_{\nu}\left(\left(\mathbf{x}_{0}, \mathbf{x}_{1}, \ldots, \mathbf{x}_{\nu}\right) \in X_{\nu}\right)\right\},
$$

where $Q_{i} \in\{\exists, \forall\}, Q_{i} \neq Q_{i+1}, \mathbf{x}_{i} \in \mathbb{R}^{n_{i}}$, and $X_{\nu}$ be either an open or a closed set in $[-1,1]^{n_{0}+\ldots+n_{\nu}}$ being a difference between a finite $C W$-complex and its subcomplex. We express an upper bound on each Betti number of $X$ via a sum of Betti numbers of some sets defined by quantifier-free formulae involving $X_{\nu}$.

In important particular cases of semialgebraic and semi-Pfaffian sets defined by quantifier-free formulae with polynomials and Pfaffian functions respectively, upper bounds on Betti numbers of $X_{\nu}$ are well known. Our results allow to extend the bounds to sets defined with quantifiers, in particular to sub-Pfaffian sets.
\end{abstract}

\section{Introduction}

Well-known results of Petrovskii, Oleinik [15], [14], Milnor [12], and Thom [18] provide an upper bound for the sum of Betti numbers of a semialgebraic set defined by a Boolean combination of polynomial equations and inequalities. A refinement of these results can be found in $[\mathbf{1}]$. For semi-Pfaffian sets the analogous bounds were obtained by Khovanskii [10] (see also [22]). In this paper we describe a reduction of estimating Betti numbers of sets defined by formulae with quantifiers to a similar problem for sets defined by a quantifier-free formulae.

More precisely, let $X$ be a subset in $[-1,1]^{n_{0}} \subset \mathbb{R}^{n_{0}}$ defined by a formula

$$
X=\left\{\mathbf{x}_{0} \mid Q_{1} \mathbf{x}_{1} Q_{2} \mathbf{x}_{2} \ldots Q_{\nu} \mathbf{x}_{\nu}\left(\left(\mathbf{x}_{0}, \mathbf{x}_{1}, \ldots, \mathbf{x}_{\nu}\right) \in X_{\nu}\right)\right\}
$$

where $Q_{i} \in\{\exists, \forall\}, Q_{i} \neq Q_{i+1}, \mathbf{x}_{i} \in \mathbb{R}^{n_{i}}$, and $X_{\nu}$ be either an open or a closed set in $[-1,1]^{n_{0}+\ldots+n_{\nu}}$ being a difference between a finite $C W$-complex and one of its subcomplexes. For instance, if $\nu=1$ and $Q_{1}=\exists$, then $X$ is the projection of $X_{\nu}$.

We express an upper bound on each Betti number of $X$ via a sum of Betti numbers of some sets defined by quantifier-free formulae involving $X_{\nu}$. In conjunction with Petrovskii-Oleinik-Thom-Milnor's result this implies a new upper bound for semialgebraic sets defined by formulae with quantifiers, which is significantly better than a bound following from the cylindrical cell decomposition approach. In conjunction with Khovanskii's result our method produces an analogous upper bound for restricted sub-Pfaffian sets defined by formulae with quantifiers. Apparently in this case no general upper bounds were previously known.

\footnotetext{
2000 Mathematics Subject Classification 14P10 (primary), 14P15, 55T99 (secondary).

The first author was supported by NSF grant \# DMS-0070666 and by James S. McDonnell Foundation. The second author was supported by the European RTN Network RAAG 20022006 (contract no HPRN-CT-0027). A part of this work was done when he was visiting Purdue University in August-September, 2001.
} 
Throughout the paper each topological space is assumed to be a difference between a finite $C W$-complex and one of its subcomplexes.

ExAmple 1. The closure $X$ of the interior of a compact set $Y \subset[-1,1]^{n}$ is homotopy equivalent to

$$
X_{\varepsilon, \delta}=\{\mathbf{x} \mid \exists \mathbf{y}(\|\mathbf{x}-\mathbf{y}\| \leq \delta) \forall \mathbf{z}(\|\mathbf{y}-\mathbf{z}\|<\varepsilon)(\mathbf{z} \in Y)\}
$$

for small enough $\delta, \varepsilon>0$ such that $\delta \gg \varepsilon$. Representing $X_{\varepsilon, \delta}$ in the form (0.1), we conclude that $X$ is homotopy equivalent to $X_{\varepsilon, \delta}=\left\{\mathbf{x} \mid \exists \mathbf{y} \forall \mathbf{z} X_{2}\right\}$, where

$$
X_{2}=\{(\mathbf{x}, \mathbf{y}, \mathbf{z}) \mid(\|\mathbf{x}-\mathbf{y}\| \leq \delta \wedge(\|\mathbf{y}-\mathbf{z}\| \geq \varepsilon \vee \mathbf{z} \in Y))\}
$$

is a closed set in $[-1,1]^{3 n}$. Our results allow to bound from above Betti numbers of $X$ in terms of Betti numbers of $X_{2}$.

\section{A spectral sequence associated with a surjective map}

Definition 1. A continuous map $f: X \rightarrow Y$ is locally split if for any $y \in Y$ there is an open neighbourhood $U$ of $y$ and a section $s: U \rightarrow X$ of $f$ (i.e., $s$ is continuous and $f s=\mathrm{Id}$ ). In particular, a projection of an open set in $\mathbb{R}^{n}$ on a subspace of $\mathbb{R}^{n}$ is always locally split.

Definition 2. For two maps $f_{1}: X_{1} \rightarrow Y$ and $f_{2}: X_{2} \rightarrow Y$, the fibered product of $X_{1}$ and $X_{2}$ is defined as

$$
X_{1} \times_{Y} X_{2}:=\left\{\left(\mathbf{x}_{1}, \mathbf{x}_{2}\right) \in X_{1} \times X_{2} \mid f_{1}\left(\mathbf{x}_{1}\right)=f_{2}\left(\mathbf{x}_{2}\right)\right\} .
$$

TheOREm 1. Let $f: X \rightarrow Y$ be a surjective cellular map. Assume that $f$ is either closed or locally split. Then for any Abelian group $G$, there exists a spectral sequence $E_{p, q}^{r}$ converging to $H_{*}(Y, G)$ with

$$
E_{p, q}^{1}=H_{q}\left(W_{p}, G\right)
$$

where

$$
W_{p}=\underbrace{X \times_{Y} \ldots \times_{Y} X}_{p+1 \text { times }}
$$

In particular,

$$
\operatorname{dim} H_{k}(Y, G) \leq \sum_{p+q=k} \operatorname{dim} H_{q}\left(W_{p}, G\right),
$$

for all $k$.

For a locally split map $f$, this theorem can be derived from [4], Corollary 1.3. We present below a proof for a closed map $f$.

REMARK 1. In the sequel we use Theorem 1 only for projections of either closed or open sets in $\mathbb{R}^{n}$. If $f$ is a projection of an open set, then (1.3) easily follows from the analogous result for closed maps which will be proved below, without references to [4]. Indeed, for an open set $Z$ define its shrinking $S(Z)$ as the closed set $Z \backslash N(\partial Z)$ where $N$ denotes an open neighbourhood. For a small enough $N(\partial Z)$, the set $Z$ is 
homotopy equivalent to $S(Z)$ (recall that $Z$ is a difference between a finite $C W-$ complex and a subcomplex). Let $X$ be open and $S(X)$ be its shrinking with a sufficiently small $N(\partial X)$. It induces shrinkings $S(Y)=f(S(X))$ and $S\left(W_{p}\right)=$ $S(X) \times_{S(Y)} \ldots \times_{S(Y)} S(X)$ which are homotopy equivalent to $Y$ and $W_{p}$ respectively. The statement for open sets $X$ and $Y$ follows from the statement for closed sets applied to $f: S(X) \rightarrow S(Y)$.

Definition 3. For a sequence $\left(P_{0}, \ldots, P_{p}\right)$ of topological spaces, their join $P_{0} *$ $\ldots * P_{p}$ can be defined as follows. Let $\Delta^{p}=\left\{s_{0} \geq 0, \ldots, s_{p} \geq 0, s_{0}+\ldots+s_{p}=1\right\}$ be the standard $p$-simplex. Then $P_{0} * \ldots * P_{p}$ is the quotient space of $P_{0} \times \ldots \times P_{p} \times \Delta^{p}$ over the following relation:

$$
\left(x_{0}, \ldots, x_{p}, s\right) \sim\left(x_{0}^{\prime}, \ldots, x_{p}^{\prime}, s\right) \text { if } s=\left(s_{0}, \ldots, s_{p}\right) \text { and } x_{i}=x_{i}^{\prime} \text { whenever } s_{i} \neq 0 .
$$

Given a continuous surjective map $f_{i}: P_{i} \rightarrow Y$ for each $i=0, \ldots, p$, the fibered join $P_{0} *_{Y} \ldots *_{Y} P_{p}$ is defined as the quotient space of $P_{0} \times_{Y} \ldots \times_{Y} P_{p} \times \Delta^{p}$ over the relation (1.4).

Definition 4. For a space $Z, 1$-st suspension of $Z$ is defined as the suspension (see $[\mathbf{1 1}])$ of $(Z \sqcup\{$ point $\})$. For an integer $p>0$, the $p$-th iteration of this operation will be called $p$-th suspension of $Z$.

Lemma 1. Let $f_{i}: P_{i} \rightarrow Y, i=0, \ldots, p$, be continuous surjective maps and $P=P_{0} *_{Y} \ldots *_{Y} P_{p}$ their fibered join. There is a natural map $F: P \rightarrow Y$ induced by the maps $f_{0}, \ldots, f_{p}$. For a point $y \in Y$ the fiber $F^{-1} y$ coincides with the join $f_{0}^{-1} y * \ldots * f_{p}^{-1} y$ of the fibers of $f_{i}$.

There is a natural map $\pi: P \rightarrow \Delta^{p}$. The fiber of $\pi$ over an interior point of $\Delta^{p}$ is $P_{0} \times_{Y} \ldots \times_{Y} P_{p}$. For each $i=0, \ldots, p$, there is a natural embedding

$$
\phi_{i}: P(i)=P_{0} *_{Y} \ldots *_{Y} P_{i-1} *_{Y} P_{i+1} *_{Y} \ldots *_{Y} P_{p} \rightarrow P .
$$

Its image coincides with $\pi^{-1}\left(\left\{s_{i}=0\right\}\right)$, and the space $P /\left(\bigcup_{i} \phi_{i}(P(i))\right)$ is homotopy equivalent to the $p$-th suspension of $P_{0} \times_{Y} \ldots \times_{Y} P_{p}$.

Proof. Directly follows from Definitions 3, 4 .

Definition 5. Let $f: X \rightarrow Y$ be a surjective continuous map. Its join space $J^{f}(X)$ is the quotient space of the disjoint union of spaces

$$
J_{p}^{f}(X)=\underbrace{X *_{Y} \ldots *_{Y} X}_{p+1 \text { times }}, \quad p=0,1, \ldots,
$$

identifying $J_{p-1}^{f}(X)$ with each of its images $\phi_{i}\left(J_{p-1}^{f}(X)\right)$ in $J_{p}^{f}(X)$ for $i=0, \ldots, p$, where $\phi_{i}$ is defined in (1.5). When $Y$ is a point, we write $J_{p}(X)$ instead of $J_{p}^{f}(X)$ and $J(X)$ instead of $J^{f}(X)$.

Lemma 2. Let $\phi: J_{p}(X) \rightarrow J(X)$ be the natural map induced by the maps $\phi_{i}$. Then $\phi\left(J_{p-1}(X)\right)$ is contractible in $\phi\left(J_{p}(X)\right)$.

Proof. Let $x$ be a point in $X$. For $t \in[0,1]$, the maps

$$
g_{t}\left(x, x_{1}, \ldots, x_{p}, s\right) \mapsto\left(x, x_{1}, \ldots, x_{p},\left(1-t+t s_{0}, t s_{1}, \ldots, t s_{p}\right)\right)
$$


define a contraction of $\phi_{0}\left(J_{p-1}(X)\right)$ to the point $x \in X$ where $X$ is identified with its embedding in $J_{p}(X)$ as $\pi^{-1}(1,0, \ldots, 0)$. It is easy to see that the maps $g_{t}$ are compatible with the equivalence relations in Definition 5 and define a contraction of $\phi\left(J_{p-1}(X)\right)$ to a point in $\phi\left(J_{p}(X)\right)$.

Lemma 3. The join space $J(X)$ is homologically trivial.

Proof. Any cycle in $J(X)$ belongs to $\phi\left(J_{p}(X)\right)$ for some $p$, while according to Lemma $2 \phi\left(J_{p}(X)\right)$ is contractible in $J(X)$. Hence the cycle is homologous to 0 . $\square$

Proof of Theorem 1. Let $f$ be closed. Let $F: J^{f}(X) \rightarrow Y$ be the natural map induced by $f$. Then $F$ is also closed. Its fiber $F^{-1} y$ over a point $y \in Y$ coincides with the join space $J\left(f^{-1} y\right)$ which is homologically trivial according to Lemma 3 . It follows that $\tilde{\bar{H}}^{*}\left(J\left(f^{-1} y\right)\right) \cong 0$, where $\bar{H}^{*}$ is the Alexander cohomology $([\mathbf{1 7}]$, p. $308)$, since $\left.\bar{H}^{*}(Z)\right) \cong H^{*}(Z)$ for any locally contractible space $Z([\mathbf{1 7}]$, p. 340), in particular for a difference between $C W$-complex and a subcomplex.

Vietoris-Begle theorem $\left([\mathbf{1 7}]\right.$, p. 344) applied to $F: J^{f}(X) \rightarrow Y$, implies

$$
\bar{H}^{*}\left(J^{f}(X), G\right) \cong \bar{H}^{*}(Y, G)
$$

and therefore

$$
H_{*}\left(J^{f}(X), G\right) \cong H_{*}(Y, G) .
$$

By Lemma 1, the space $J_{p}^{f}(X) /\left(\bigcup_{q<p} J_{q}^{f}(X)\right)$ is homotopy equivalent to the $p$-th suspension of $W_{p}$. Theorem 1 follows now from the spectral sequence associated with filtration of $J^{f}(X)$ by the spaces $J_{p}^{f}(X)$.

REMARK 2. For a map $f$ with 0-dimensional fibers, a similar spectral sequence, "image computing spectral sequence" was applied to problems in theory of singularities and topology by Vassiliev [19], Goryunov-Mond [7], Goryunov [6], Houston $[\mathbf{9}]$, and others.

Remark 3. A continuous map $f: X \rightarrow Y$ is called compact-covering if any compact set in $Y$ is an image of a compact set in $X$. This condition includes both the closed and the locally split cases and may be more convenient for applications. For a compact-covering $f: X \rightarrow Y$ Theorem 1 is also true. A proof will appear elsewhere.

\section{Alexander's duality and Mayer-Vietoris inequality}

Let

$$
I_{i}^{n}:=\bigcap_{1 \leq j \leq n}\left\{-i \leq x_{j} \leq i\right\} \subset \mathbb{R}^{n}
$$

Define the "thick boundary" $\partial I_{i}^{n}:=I_{i+1}^{n} \backslash I_{i}^{n}$. The following lemma is a version of Alexander's duality theorem.

Lemma 4. (Alexander's duality) If $X \subset I_{i}^{n}$ is an open set in $I_{i}^{n}$, then for any $q \in \mathbb{Z}, q \leq n-1$,

$$
H_{q}\left(I_{i}^{n} \backslash X, \mathbb{R}\right) \cong \tilde{H}_{n-q-1}\left(X \cup \partial I_{i}^{n}, \mathbb{R}\right)
$$


If $X \subset I_{i}^{n}$ is a closed set in $I_{i}^{n}$, then for any $q \in \mathbb{Z}, q \leq n-1$,

$$
H_{q}\left(I_{i}^{n} \backslash X, \mathbb{R}\right) \cong \tilde{H}_{n-q-1}\left(X \cup \operatorname{closure}\left(\partial I_{i}^{n}\right), \mathbb{R}\right) .
$$

Proof. For definiteness let $X$ be closed. Compactifying $\mathbb{R}^{n}$ at infinity as $\mathbb{R}^{n} \cup$ $\infty \simeq S^{n}$, we have, by Alexander's duality [11],

$$
\tilde{H}_{q}\left(S ^ { n } \backslash ( X \cup \operatorname { c l o s u r e } ( \partial I _ { i } ^ { n } ) , \mathbb { R } ) \cong \tilde { H } _ { n - q - 1 } \left(\left(X \cup \operatorname{closure}\left(\partial I_{i}^{n}\right), \mathbb{R}\right) .\right.\right.
$$

The first group is isomorphic to $H_{q}\left(I_{i}^{n} \backslash X, \mathbb{R}\right)$ when $q>0$, and to $\tilde{H}_{0}\left(I_{i}^{n} \backslash X, \mathbb{R}\right)+\mathbb{R} \cong$ $H_{0}\left(I_{i}^{n} \backslash X, \mathbb{R}\right)$ when $q=0$. Combining these two cases, we obtain (2.2).

Lemma 5. (Mayer-Vietoris inequality) Let $X_{1}, \ldots, X_{m} \subset I_{1}^{n}$ be all open or all closed in $I_{1}^{n}$. Then

$$
\mathrm{b}_{i}\left(\bigcup_{1 \leq j \leq n} X_{j}\right) \leq \sum_{J \subset\{1, \ldots, n\}} \mathrm{b}_{i-|J|+1}\left(\bigcap_{j \in J} X_{j}\right)
$$

and

$$
\mathrm{b}_{i}\left(\bigcap_{1 \leq j \leq n} X_{j}\right) \leq \sum_{J \subset\{1, \ldots, n\}} \mathrm{b}_{i+|J|-1}\left(\bigcup_{j \in J} X_{j}\right)
$$

where $\mathrm{b}_{i}$ is the ith Betti number.

Proof. A well-known corollary to Mayer-Vietoris sequence.

\section{Thom-Milnor's and Khovanskii's bounds}

Necessary definitions regarding semi-Pfaffian and sub-Pfaffian sets can be found in $[\mathbf{1 0}]$, [5]. In this paper we consider only restricted sub-Pfaffian sets.

To apply our results to semialgebraic sets and to restricted sub-Pfaffian sets, defined by formulae with quantifiers, we need the following known upper bounds on Betti numbers for sets defined by quantifier-free formulae.

Let $X=\{\varphi\} \subset I_{1}^{n}$ be a semialgebraic set, where $\varphi$ is a Boolean combination with no negations of $s$ atomic formulae of the kind $f>0, f$ being polynomials in $n$ variables with coefficients in $\mathbb{R}, \operatorname{deg}(f)<d$. We will refer to the sequence $(n, s, d)$ as to format of $\varphi$. It follows from [18], [12], [1] that the sum of Betti numbers of $X$ is

$$
\mathrm{b}(X) \leq O(s d)^{n} .
$$

If $X=\{\varphi\}$ is a compact semialgebraic set, where $\varphi$ is a Boolean combination with no negations of $s$ atomic formulae of the kind either $f \geq 0$ or $f>0, f$ being polynomials in $n$ variables, $\operatorname{deg}(f)<d$, then a combination of results from [18], $[\mathbf{1 2}],[\mathbf{1}]$ and $[\mathbf{1 3}],[\mathbf{2 1}]$ implies that that the sum of Betti numbers of $X$ also satisfies (3.1).

Now let $X=\{\varphi\} \subset I_{1}^{n}$ be a semi-Pfaffian set, where $\varphi$ is a Boolean combination with no negations of $s$ atomic formulae of the kind $f>0, f$ being Pfaffian functions in an open domain $G \supset I_{1}^{n}$ of order $\rho$, degree $(\alpha, \beta)$, having a common Pfaffian chain with coefficients in $\mathbb{R}$. The sequence $(n, s, \alpha, \beta, \rho)$ is called format of $\varphi$. It follows from $[\mathbf{1 0}],[\mathbf{2 2}]$ that the sum of Betti numbers of $X$ is

$$
\mathrm{b}(X) \leq s^{n} 2^{\rho(\rho-1) / 2} O(n \beta+\min \{n, \rho\} \alpha)^{n+\rho} .
$$


Let $X \subset I_{1}^{n_{0}}$ be a semialgebraic set defined by a formula

$$
Q_{1} \mathbf{x}_{1} Q_{2} \mathbf{x}_{2} \ldots Q_{\nu} \mathbf{x}_{\nu} F\left(\mathbf{x}_{0}, \mathbf{x}_{1}, \ldots \mathbf{x}_{\nu}\right)
$$

where $Q_{i} \in\{\exists, \forall\}, Q_{i} \neq Q_{i+1}, \mathbf{x}_{i}=\left(x_{i, 1}, \ldots, x_{i, n_{i}}\right) \in I_{1}^{n_{i}}$, and $F$ is a quantifierfree Boolean formula with no negations having $s$ atoms of the kind $f>0$, where $f$ 's are polynomials with real coefficients of degrees less than $d$. The cylindrical algebraic decomposition technique from $[\mathbf{3}],[\mathbf{2 0}]$ allows to bound from above the number of cells in a representation of $X$ as a difference between a $C W$-complex and its subcomplex. In particular,

$$
\mathrm{b}(X) \leq(s d)^{2^{O(n)}} .
$$

A better upper bound can be obtained as follows. According to $[\mathbf{2}]$ (which refines $[\mathbf{8}],[\mathbf{1 6}])$, there exists a Boolean combination

$$
\psi\left(\mathbf{x}_{0}\right)=\bigvee_{1 \leq i \leq I} \bigwedge_{1 \leq j \leq J_{i}}\left(g_{i, j}\left(\mathbf{x}_{0}\right) *_{i, j} 0\right),
$$

such that $X=\left\{\psi\left(\mathbf{x}_{0}\right)\right\}$. Here

$$
\begin{gathered}
*_{i, j} \in\{=,<,>\}, \quad g_{i, j} \in \mathbb{R}\left[\mathbf{x}_{0}\right], \quad \operatorname{deg}\left(g_{i, j}\right)<d^{\prod_{i \geq 1} O\left(n_{i}\right)}, \\
I<s^{\left(n_{0}+1\right) \prod_{i \geq 1}\left(n_{i}+1\right)} d^{\left(n_{0}+1\right) \prod_{i \geq 1} O\left(n_{i}\right)}, \\
J_{i}<s^{\prod_{i \geq 1}\left(n_{i}+1\right)} d^{\prod_{i \geq 1} O\left(n_{i}\right)} .
\end{gathered}
$$

Applying (3.1) to $X=\left\{\psi\left(\mathbf{x}_{0}\right)\right\}$, we get

$$
\mathrm{b}\left(X_{0}\right) \leq s^{O\left(n_{0}^{2} \prod_{i \geq 1} n_{i}\right)} d^{O\left(n_{0}^{2}\right) \prod_{i \geq 1} O\left(n_{i}\right)} \leq(s d)^{O\left(n_{0}^{2}\right) \prod_{i \geq 1} O\left(n_{i}\right)}
$$

\section{Basic notation}

Let $X=\widetilde{X_{0}}=I_{1}^{n_{0}} \backslash X_{0} \subset I_{1}^{n_{0}}$ be a set defined by a formula (0.1). For example, $X$ could be a sub-Pfaffian or a semialgebraic set defined by (3.3), where $F$ is a quantifier-free Boolean formula with no negations. For definiteness assume that $Q_{1}=\exists$ and $X$ is open in $I_{1}^{n_{0}}$.

Define

$$
X_{i}:=\left\{\left(\mathbf{x}_{0}, \ldots, \mathbf{x}_{i}\right) \mid Q_{i+1} \mathbf{x}_{i+1} Q_{i+2} \mathbf{x}_{i+2} \ldots Q_{\nu} \mathbf{x}_{\nu}\left(\left(\mathbf{x}_{0}, \mathbf{x}_{1}, \ldots, \mathbf{x}_{\nu}\right) \in X_{\nu}\right)\right\}
$$

for odd $i$ and

$$
X_{i}:=I_{1}^{n_{0}+\ldots+n_{i}} \backslash\left\{\left(\mathbf{x}_{0}, \ldots, \mathbf{x}_{i}\right) \mid Q_{i+1} \mathbf{x}_{i+1} Q_{i+2} \mathbf{x}_{i+2} \ldots Q_{\nu} \mathbf{x}_{\nu}\left(\left(\mathbf{x}_{0}, \mathbf{x}_{1}, \ldots, \mathbf{x}_{\nu}\right) \in X_{\nu}\right)\right\}
$$

for even $i$. Then $\pi_{i}\left(X_{i}\right)=\widetilde{X_{i-1}}$, where $\pi_{i}: \mathbb{R}^{n_{0}+\ldots+n_{i}} \rightarrow \mathbb{R}^{n_{0}+\ldots+n_{i-1}}$ and tilde denotes the complement in $I_{1}^{n_{0}+\ldots+n_{i-1}}$.

For a set $I_{i}^{m_{i}} \times I_{i-1}^{m_{i-1}} \times \ldots \times I_{1}^{m_{1}}$ define $\partial\left(I_{i}^{m_{i}} \times I_{i-1}^{m_{i-1}} \times \ldots \times I_{1}^{m_{1}}\right)$ as

$$
\left(I_{i+1}^{m_{i}} \times I_{i}^{m_{i-1}} \times \ldots \times I_{2}^{m_{1}}\right) \backslash\left(I_{i}^{m_{i}} \times I_{i-1}^{m_{i-1}} \times \ldots \times I_{1}^{m_{1}}\right)
$$

for even $i$ and as the closure of this difference for odd $i$.

Let $p_{1}, \ldots, p_{i}$ be some positive integers to be specified later. Define

$$
B_{i}^{i}:=\partial\left(I_{i-1}^{n_{0}+\left(p_{1}+1\right) n_{1}} \times I_{i-2}^{\left(p_{2}+1\right) n_{2}} \times \ldots \times I_{1}^{\left(p_{i-1}+1\right) n_{i-1}}\right) \times I_{1}^{n_{i}} .
$$

For any $j, i<j \leq \nu$ define $B_{j}^{i}:=\widetilde{B_{j-1}^{i}} \times I_{1}^{n_{j}}$, where tilde denotes the complement in the appropriate cube. 


\section{DEFINITION 6.}

(i) Let $Y \subset I_{v}^{n_{0}} \times I_{v}^{\left(p_{1}+1\right) n_{1}} \times I_{v-1}^{\left(p_{2}+1\right) n_{2}} \times \ldots \times I_{v-l+2}^{\left(p_{l-1}+1\right) n_{l-1}} \times I_{1}^{n_{l}+\ldots+n_{i}}$, where $1 \leq l \leq i, v \geq i$, and let $J \subset\left\{\left(j_{l}, \ldots, j_{i}\right) \mid 1 \leq j_{k} \leq p_{k}+1, l \leq k \leq i\right\}$. Then define $\prod_{i, J}^{l} Y$ as an intersection of sets

$$
\begin{gathered}
\left\{\left(\mathbf{x}_{0}, \mathbf{x}_{1}^{(1)}, \ldots, \mathbf{x}_{1}^{\left(p_{1}+1\right)}, \ldots, \mathbf{x}_{i}^{(1)}, \ldots, \mathbf{x}_{i}^{\left(p_{i}+1\right)}\right) \mid\right. \\
\mathbf{x}_{0} \in I_{v}^{n_{0}}, \mathbf{x}_{k}^{(m)} \in I_{v-k+1}^{n_{k}}(1 \leq k \leq l-1), \\
\left.\mathbf{x}_{k}^{(m)} \in I_{1}^{n_{k}}(l \leq k \leq i),\left(\mathbf{x}_{0}, \mathbf{x}_{1}^{(1)}, \ldots, \mathbf{x}_{l-1}^{\left(p_{l-1}+1\right)}, \mathbf{x}_{l}^{\left(j_{l}\right)}, \ldots, \mathbf{x}_{i}^{\left(j_{i}\right)}\right) \in Y\right\}
\end{gathered}
$$

over all $\left(j_{l}, \ldots, j_{i}\right) \in J$.

(ii) Let $Y \subset I_{v}^{n_{0}} \times I_{v}^{\left(p_{1}+1\right) n_{1}} \times I_{v-1}^{\left(p_{2}+1\right) n_{2}} \times \ldots \times I_{v-l+2}^{\left(p_{l-1}+1\right) n_{l-1}} \times I_{1}^{n_{l}+\ldots+n_{i}+n_{i+1}}$. Define $\prod_{i, J}^{l, i+1} Y$ as an intersection of sets

$$
\begin{gathered}
\left\{\left(\mathbf{x}_{0}, \mathbf{x}_{1}^{(1)}, \ldots, \mathbf{x}_{1}^{\left(p_{1}+1\right)}, \ldots, \mathbf{x}_{i}^{(1)}, \ldots, \mathbf{x}_{i}^{\left(p_{i}+1\right)}, \mathbf{x}_{i+1}\right) \mid\right. \\
\mathbf{x}_{0} \in I_{v}^{n_{0}}, \mathbf{x}_{k}^{(m)} \in I_{v-k+1}^{n_{k}}(1 \leq k \leq l-1), \mathbf{x}_{k}^{(m)} \in I_{1}^{n_{k}}(l \leq k \leq i), \mathbf{x}_{i+1} \in I_{1}^{n_{i+1}}, \\
\left.\left(\mathbf{x}_{0}, \mathbf{x}_{1}^{(1)}, \ldots, \mathbf{x}_{l-1}^{\left(p_{l-1}+1\right)}, \mathbf{x}_{l}^{\left(j_{l}\right)}, \ldots, \mathbf{x}_{i}^{\left(j_{i}\right)}, \mathbf{x}_{i+1}\right) \in Y\right\}
\end{gathered}
$$

over all $\left(j_{l}, \ldots, j_{i}\right) \in J$.

(iii) If $l=i$ and $J=\left\{j \mid 1 \leq j \leq p_{i}+1\right\}$ we use the notation $\prod_{i}^{i} Y$ for $\prod_{i, J}^{i} Y$.

Lemma 6. Let

$$
Y \subset I_{v}^{n_{0}} \times I_{v}^{\left(p_{1}+1\right) n_{1}} \times I_{v-1}^{\left(p_{2}+1\right) n_{2}} \times \ldots \times I_{v-l+2}^{\left(p_{l-1}+1\right) n_{l-1}} \times I_{1}^{n_{l}+\ldots+n_{i}+n_{i+1}} .
$$

Then for any $J \subset\left\{j \mid 1 \leq j \leq p_{i+1}+1\right\}, J^{\prime} \subset\left\{\left(j_{l}, \ldots, j_{i}\right) \mid 1 \leq j_{k} \leq p_{k}+1, l \leq k \leq i\right\}$ we have

$$
\prod_{i+1, J}^{i+1} \prod_{i, J^{\prime}}^{l, i+1} Y=\prod_{i+1, J^{\prime} \times J}^{l} Y .
$$

Proof. Straightforward.

Definition 7. Let $Y, l, i, J$ be as in Definition 6. Define $\bigsqcup_{i, J}^{l} Y$ and $\bigsqcup_{i, J}^{l, i+1} Y$ similar to $\prod_{i, J}^{l} Y$ and $\prod_{i, J}^{l, i+1} Y$ respectively, replacing in Definition 6 "intersection" by "union".

Lemma 7. (De Morgan law)

$$
\begin{aligned}
\bigsqcup_{i, J}^{l} Y & =\left(\prod_{i, J}^{l} \tilde{Y}\right)^{\tilde{j}} \\
\bigsqcup_{i, J}^{l, i+1} Y & =\left(\prod_{i, J}^{l, i+1} \tilde{Y}\right)^{\sim},
\end{aligned}
$$

where tildes denote complements in the appropriate cubes.

Proof. Straightforward.

Definition 8. Let $t_{i}=n_{0}+n_{1}\left(p_{1}+1\right)+\ldots+n_{i}\left(p_{i}+1\right)$. Define projection maps

$$
\pi_{i}: \mathbb{R}^{n_{0}+\ldots+n_{i}} \rightarrow \mathbb{R}^{n_{0}+\ldots+n_{i-1}}
$$




$$
\left(\mathbf{x}_{0}, \ldots, \mathbf{x}_{i}\right) \mapsto\left(\mathbf{x}_{0}, \ldots, \mathbf{x}_{i-1}\right),
$$

and for $j<i$,

$$
\begin{gathered}
\pi_{i, j}: \mathbb{R}^{t_{j}+n_{j+1}+\ldots+n_{i}} \rightarrow \mathbb{R}^{t_{j}+n_{j+1}+\ldots+n_{i-1}} \\
\left(\mathbf{x}_{0}, \mathbf{x}_{1}^{(1)}, \ldots, \mathbf{x}_{j}^{\left(p_{j}+1\right)}, \mathbf{x}_{j+1}, \ldots, \mathbf{x}_{i}\right) \mapsto\left(\mathbf{x}_{0}, \mathbf{x}_{1}^{(1)}, \ldots, \mathbf{x}_{j}^{\left(p_{j}+1\right)}, \mathbf{x}_{j+1}, \ldots, \mathbf{x}_{i-1}\right) .
\end{gathered}
$$

LEMma 8. Let

$$
Y \subset I_{v}^{n_{0}} \times I_{v}^{\left(p_{1}+1\right) n_{1}} \times I_{v-1}^{\left(p_{2}+1\right) n_{2}} \times \ldots \times I_{v-l+2}^{\left(p_{l-1}+1\right) n_{l-1}} \times I_{1}^{n_{l}+\ldots+n_{i}+n_{i+1}} .
$$

Then

$$
\bigsqcup_{i, J}^{l} \pi_{i+1, l-1}(Y)=\pi_{i+1, i}\left(\bigsqcup_{i, J}^{l, i+1} Y\right) .
$$

Proof. Straightforward.

5. Case of a single quantifier block

According to Theorem 1,

$$
\mathrm{b}_{q_{0}}(X)=\mathrm{b}_{q_{0}}\left(\widetilde{X_{0}}\right) \leq \sum_{p_{1}+q_{1}=q_{0}} \mathrm{~b}_{q_{1}}\left(\prod_{1, J_{1}^{1}}^{1} X_{1}\right),
$$

where $J_{1}^{1}=\left\{1, \ldots, p_{1}+1\right\}$.

Let $\nu=1$, then (3.3) turns into $\exists \mathbf{x}_{1} F\left(\mathbf{x}_{0}, \mathbf{x}_{1}\right)$, where $X_{1}=\left\{F\left(\mathbf{x}_{0}, \mathbf{x}_{1}\right)\right\}$ and $F\left(\mathbf{x}_{0}, \mathbf{x}_{1}\right)$ is a Boolean combination with no negations of $s$ atomic formulae of the kind $f>0$.

\subsection{Polynomial case}

Suppose that $X_{1}$ is semialgebraic, with $f$ 's being polynomials of degrees $\operatorname{deg}(f)<$ $d$. For any $k \leq \operatorname{dim}(X)$, we bound the Betti number $\mathrm{b}_{k}(X)$ from above in the following way. Observe that $\prod_{1, J_{1}^{1}}^{1} X_{1}$ is an open set in $I_{1}^{n_{0}+\left(p_{1}+1\right) n_{1}}$ definable by a Boolean combination with no negations of $\left(p_{1}+1\right) s$ atomic formulae of the kind $g>0, \operatorname{deg}(g)<d$ in $t_{1}=n_{0}+\left(p_{1}+1\right) n_{1}$ variables.

According to (3.1), for any $q_{1} \leq \operatorname{dim}(X)$,

$$
\mathrm{b}_{q_{1}}\left(\prod_{1, J_{1}^{1}}^{1} X_{1}\right) \leq O\left(p_{1} s d\right)^{n_{0}+\left(p_{1}+1\right) n_{1}} .
$$

Then due to (5.1), for any $k \leq \operatorname{dim}(X) \leq n_{0}$,

$$
\mathrm{b}_{k}(X) \leq \sum_{p_{1}+q_{1}=k} O\left(p_{1} s d\right)^{n_{0}+\left(p_{1}+1\right) n_{1}} \leq(k s d)^{O\left(n_{0}+k n_{1}\right)} .
$$

\subsection{Pfaffian case}

Suppose that $X_{1} \subset I_{1}^{n}$ is sub-Pfaffian, with $f$ 's being Pfaffian functions in an open domain $G \supset I_{1}^{n}$ of order $\rho$, degree $(\alpha, \beta)$, having a common Pfaffian chain. Observe that $\prod_{1, J_{1}^{1}}^{1} X_{1}$ is an open set definable by a Boolean combination with no negations of $\left(p_{1}+1\right) s$ atomic formulae of the kind $g>0$, where $g$ are Pfaffian functions in an open domain contained in $I_{1}^{n_{0}+\left(p_{1}+1\right) n_{1}}$ of degrees $(\alpha, \beta)$, order $\left(p_{1}+1\right) \rho$ in $n_{0}+\left(p_{1}+1\right) n_{1}$ variables, having a common Pfaffian chain. According to (3.2), for 
any $q_{1} \leq \operatorname{dim}(X)$,

$$
\begin{gathered}
\mathrm{b}_{q_{1}}\left(\prod_{1, J_{1}^{1}}^{1} X_{1}\right) \leq\left(\left(p_{1}+1\right) s\right)^{n_{0}+\left(p_{1}+1\right) n_{1}} 2^{\left(p_{1}+1\right) \rho\left(\left(p_{1}+1\right) \rho-1\right) / 2} . \\
\cdot O\left(\left(n_{0}+p_{1} n_{1}\right) \beta+\min \left\{p_{1} \rho, n_{0}+p_{1} n_{1}\right\} \alpha\right)^{n_{0}+\left(p_{1}+1\right)\left(n_{1}+\rho\right)} .
\end{gathered}
$$

Then due to (5.1), for any $k \leq \operatorname{dim}(X) \leq n_{0}$,

$$
\begin{gathered}
\mathrm{b}_{k}(X) \leq \sum_{p_{1}+q_{1}=k} \mathrm{~b}_{q_{1}}\left(\prod_{1, J_{1}^{1}}^{1} X_{1}\right) \leq \\
k((k+1) s)^{n_{0}+(k+1) n_{1}} 2^{(k+1) \rho((k+1) \rho-1) / 2} . \\
O\left(\left(n_{0}+k n_{1}\right) \beta+\min \left\{k \rho, n_{0}+k n_{1}\right\} \alpha\right)^{n_{0}+(k+1)\left(n_{1}+\rho\right)} .
\end{gathered}
$$

Let $d>\alpha+\beta$. Relaxing the obtained bound, we get

$$
\mathrm{b}_{k}(X) \leq(k s)^{O\left(n_{0}+k n_{1}\right)} 2^{(O(k \rho))^{2}}\left(\left(n_{0}+k n_{1}\right) d\right)^{O\left(n_{0}+k n_{1}+k \rho\right)} .
$$

\section{Cases of two and three quantifier blocks}

In this section we obtain a generalization of (5.1) to the case of two and three blocks of quantifiers, as a preparation for cumbersome general formulae in the next section. The case of three quantifier blocks is considered separately also because of a technical difficulty that appears first in that case (see the discussion after (6.1)).

Recall that

$$
\pi_{i}: \mathbb{R}^{n_{0}+\ldots+n_{i}} \rightarrow \mathbb{R}^{n_{0}+\ldots+n_{i-1}},
$$

for $j<i$,

$$
\pi_{i, j}: \mathbb{R}^{t_{j}+n_{j+1}+\ldots+n_{i}} \rightarrow \mathbb{R}^{t_{j}+n_{j+1}+\ldots+n_{i-1}} .
$$

Let $\nu=3$, then the original formula becomes $\exists \mathbf{x}_{1} \forall \mathbf{x}_{2} \exists \mathbf{x}_{3}\left(\left(\mathbf{x}_{0}, \mathbf{x}_{1}, \mathbf{x}_{2}, \mathbf{x}_{3}\right) \in X_{3}\right)$. Thereby,

$$
X_{1}=\left\{\forall \mathbf{x}_{2} \exists \mathbf{x}_{3}\left(\left(\mathbf{x}_{0}, \mathbf{x}_{1}, \mathbf{x}_{2}, \mathbf{x}_{3}\right) \in X_{3}\right)\right\}, \widetilde{X_{2}}=\left\{\exists \mathbf{x}_{3}\left(\left(\mathbf{x}_{0}, \mathbf{x}_{1}, \mathbf{x}_{2}, \mathbf{x}_{3}\right) \in X_{3}\right)\right\},
$$

$X=\widetilde{X_{0}}$ is open in $I_{1}^{n_{0}}$.

According to Theorem 1 ,

$$
\mathrm{b}_{q_{0}}\left(\widetilde{X_{0}}\right) \leq \sum_{p_{1}+q_{1}=q_{0}} \mathrm{~b}_{q_{1}}\left(\prod_{1, J_{1}^{1}}^{1} X_{1}\right)
$$

Applying in succession Lemma 7 (De Morgan law), Lemma 4 (Alexander's duality), definitions of $\pi_{2}$ and $\pi_{2,1}$, and Lemma 8 we get

$$
\begin{gathered}
\mathrm{b}_{q_{1}}\left(\prod_{1, J_{1}^{1}}^{1} X_{1}\right)=\mathrm{b}_{q_{1}}\left(\left(\bigsqcup_{1, J_{1}^{1}}^{1} \widetilde{X_{1}}\right)\right) \leq \\
\leq \mathrm{b}_{t_{1}-q_{1}-1}\left(\bigsqcup_{1, J_{1}^{1}}^{1} \widetilde{X_{1}} \cup \partial I_{1}^{t_{1}}\right)=\mathrm{b}_{t_{1}-q_{1}-1}\left(\bigsqcup_{1, J_{1}^{1}}^{1} \pi_{2}\left(X_{2}\right) \cup \pi_{2,1}\left(\partial I_{1}^{t_{1}} \times I_{1}^{n_{2}}\right)\right)= \\
=\mathrm{b}_{t_{1}-q_{1}-1}\left(\pi_{2,1}\left(\bigsqcup_{1, J_{1}^{1}}^{1,2} X_{2} \cup \partial I_{1}^{t_{1}} \times I_{1}^{n_{2}}\right)\right) .
\end{gathered}
$$

Due to Theorem 1, the last expression does not exceed

$$
\sum_{p_{2}+q_{2}=t_{1}-q_{1}-1} \mathrm{~b}_{q_{2}}\left(\prod_{2}^{2}\left(\bigsqcup_{1, J_{1}^{1}}^{1,2} X_{2} \cup \partial I_{1}^{t_{1}} \times I_{1}^{n_{2}}\right)\right)=
$$




$$
=\sum_{p_{2}+q_{2}=t_{1}-q_{1}-1} \mathrm{~b}_{q_{2}}\left(\prod_{2}^{2}\left(\bigsqcup_{1, J_{1}^{1}}^{1,2} X_{2} \cup B_{2}^{2}\right)\right) \text {. }
$$

In a case of sub-Pfaffian or semialgebraic $X$ it is now possible to estimate

$$
\mathrm{b}_{q_{2}}\left(\prod_{2}^{2}\left(\bigsqcup_{1, J_{1}^{1}}^{1,2} X_{2} \cup B_{2}^{2}\right)\right)
$$

via the format of $X_{2}$. This completes the description of the case of two quantifier blocks. We now proceed to the case of three blocks.

Due to Lemma 7 (De Morgan law) and Lemma 4 (Alexander's duality),

$$
\begin{array}{r}
\mathrm{b}_{q_{2}}\left(\prod_{2}^{2}\left(\bigsqcup_{1, J_{1}^{1}}^{1,2} X_{2} \cup B_{2}^{2}\right)\right)=\mathrm{b}_{q_{2}}\left(\left(\bigsqcup_{2}^{2}\left(\prod_{1, J_{1}^{1}}^{1,2} \widetilde{X_{2}} \cap \widetilde{B_{2}^{2}}\right)\right)\right)= \\
=\mathrm{b}_{t_{2}-q_{2}-1}\left(\bigsqcup_{2}^{2}\left(\prod_{1, J_{1}^{1}}^{1,2} \widetilde{X_{2}} \cap \widetilde{B_{2}^{2}}\right) \cup \partial\left(I_{2}^{t_{1}} \times I_{1}^{n_{2}\left(p_{2}+1\right)}\right)\right) .
\end{array}
$$

From this point we could have proceeded in a "natural" way similar to the just considered case of two blocks, namely, replacing in the previous expression the set $\widetilde{X_{2}}$ by $\pi_{3}\left(X_{3}\right)$, then carrying the projection operator to the left to obtain an expression of the kind $\mathrm{b}_{t_{2}-q_{2}-1}\left(\pi_{3,2}(\ldots)\right)$, and after that applying Theorem 1 . However, carrying the projection operator through the symbol $\prod_{1, J_{1}^{1}}^{1,2}$ (which corresponds to an intersection of some cylindrical sets) would require an introduction of $p_{1} n_{2}$ new variables. This would result in a significantly higher upper bound for $\mathrm{b}_{q_{0}}(X)$. Instead we reduce intersections to unions, then carrying the projection operator to the left does not require new variables.

More precisely, by Lemma 5 (Mayer-Vietoris inequality) expression (6.1) does not exceed

$$
\begin{gathered}
\sum_{1 \leq k_{2} \leq p_{2}+1} \sum_{\substack{\hat{J}_{2}^{2} \subset\left\{1, \ldots, p_{2}+1\right\},\left|\hat{J}_{2}^{2}\right|=k_{2}}} \\
\mathrm{~b}_{t_{2}-q_{2}-k_{2}}\left(\prod_{2, \hat{J}_{2}^{2}}^{2}\left(\prod_{1, J_{1}^{1}}^{1,2} \widetilde{X_{2}} \cap \widetilde{B_{2}^{2}}\right) \cup \partial\left(I_{2}^{t_{1}} \times I_{1}^{n_{2}\left(p_{2}+1\right)}\right)\right) .
\end{gathered}
$$

(We estimate a Betti number of the union of cylindrical sets from the definition of the symbol $\bigsqcup_{2}^{2}$ by a sum of Betti numbers of intersections of various combinations of these sets.)

By Lemma 6,

$$
\begin{gathered}
\mathrm{b}_{t_{2}-q_{2}-k_{2}}\left(\prod_{2, \hat{J}_{2}^{2}}^{2}\left(\prod_{1, J_{1}^{1}}^{1,2} \widetilde{X_{2}} \cap \widetilde{B_{2}^{2}}\right) \cup \partial\left(I_{2}^{t_{1}} \times I_{1}^{n_{2}\left(p_{2}+1\right)}\right)\right)= \\
=\mathrm{b}_{t_{2}-q_{2}-k_{2}}\left(\prod_{2, J_{1}^{1} \times \hat{J}_{2}^{2}}^{1} \widetilde{X_{2}} \cap \prod_{2, J_{1}^{2} \times \hat{J}_{2}^{2}}^{2} \widetilde{B_{2}^{2}} \cup \partial\left(I_{2}^{t_{1}} \times I_{1}^{n_{2}\left(p_{2}+1\right)}\right)\right),
\end{gathered}
$$

with $J_{1}^{2}=\{1\}$. By Lemma 5 (Mayer-Vietoris inequality) the last expression does not exceed

$$
\begin{gathered}
\sum_{1 \leq s_{2} \leq q_{2}+k_{2}+1} \sum_{J_{2}^{1} \subset J_{1}^{1} \times \hat{J}_{2}^{2},} \sum_{J_{2}^{2} \subset J_{1}^{2} \times \hat{J}_{2}^{2},\left|J_{2}^{1}\right|+\left|J_{2}^{2}\right|=s_{2}} \\
\mathrm{~b}_{t_{2}-q_{2}-k_{2}+s_{2}-1}\left(\bigsqcup_{2, J_{2}^{1}}^{1} \widetilde{X_{2}} \cup \bigsqcup_{2, J_{2}^{2}}^{2} \widetilde{B_{2}^{2}} \cup \partial\left(I_{2}^{t_{1}} \times I_{1}^{n_{2}\left(p_{2}+1\right)}\right)\right),
\end{gathered}
$$

taking into the account that

$$
\operatorname{dim}\left(\bigsqcup_{2, J_{2}^{1}}^{1} \widetilde{X_{2}} \cup \bigsqcup_{2, J_{2}^{2}}^{2} \widetilde{B_{2}^{2}} \cup \partial\left(I_{2}^{t_{1}} \times I_{1}^{n_{2}\left(p_{2}+1\right)}\right)\right) \leq t_{2}
$$


and therefore

$$
\mathrm{b}_{t_{2}-q_{2}-k_{2}+s_{2}-1}\left(\bigsqcup_{2, J_{2}^{1}}^{1} \widetilde{X_{2}} \cup \bigsqcup_{2, J_{2}^{2}}^{2} \widetilde{B_{2}^{2}} \cup \partial\left(I_{2}^{t_{1}} \times I_{1}^{n_{2}\left(p_{2}+1\right)}\right)\right)=0
$$

for $s_{2}>q_{2}+k_{2}+1$.

We have

$$
\begin{gathered}
\mathrm{b}_{t_{2}-q_{2}-k_{2}+s_{2}-1}\left(\bigsqcup_{2, J_{2}^{1}}^{1} \widetilde{X_{2}} \cup \bigsqcup_{2, J_{2}^{2}}^{2} \widetilde{B_{2}^{2}} \cup \partial\left(I_{2}^{t_{1}} \times I_{1}^{n_{2}\left(p_{2}+1\right)}\right)\right)= \\
=\mathrm{b}_{t_{2}-q_{2}-k_{2}+s_{2}-1}\left(\bigsqcup_{2, J_{2}^{1}}^{1} \pi_{3}\left(X_{3}\right) \cup \bigsqcup_{2, J_{2}^{2}}^{2} \pi_{3,1}\left(B_{3}^{2}\right) \cup \pi_{3,2}\left(\partial\left(I_{2}^{t_{1}} \times I_{1}^{n_{2}\left(p_{2}+1\right)}\right) \times I_{1}^{n_{3}}\right)\right)= \\
=\mathrm{b}_{t_{2}-q_{2}-k_{2}+s_{2}-1}\left(\pi_{3,2}\left(\bigsqcup_{2, J_{2}^{1}}^{1,3} X_{3} \cup \bigsqcup_{2, J_{2}^{2}}^{2,3} B_{3}^{2} \cup B_{3}^{3}\right)\right) .
\end{gathered}
$$

Due to Theorem 1 the last expression does not exceed

$$
\sum_{p_{3}+q_{3}=t_{2}-q_{2}-k_{2}+s_{2}-1} \mathrm{~b}_{q_{3}}\left(\prod_{3}^{3}\left(\bigsqcup_{2, J_{2}^{1}}^{1,3} X_{3} \cup \bigsqcup_{2, J_{2}^{2}}^{2,3} B_{3}^{2} \cup B_{3}^{3}\right)\right) .
$$

In case of a sub-Pfaffian or a semialgebraic $X$ it is now possible to estimate

$$
\mathrm{b}_{q_{3}}\left(\prod_{3}^{3}\left(\bigsqcup_{2, J_{2}^{1}}^{1,3} X_{3} \cup \bigsqcup_{2, J_{2}^{2}}^{2,3} B_{3}^{2} \cup B_{3}^{3}\right)\right)
$$

via the format of $X_{3}$.

\section{Arbitrary number of quantifiers}

TheOREm 2. For any $i$ the Betti number $\mathrm{b}_{q_{0}}(X)$ does not exceed

$$
\begin{aligned}
& \sum_{p_{1}+q_{1}=q_{0}} \sum_{p_{2}+q_{2}=t_{1}-q_{1}-1} \sum_{1 \leq k_{2} \leq p_{2}+1} \sum_{\hat{J}_{2}^{2} \subset\left\{1, \ldots, p_{2}+1\right\},\left|\hat{J}_{2}^{2}\right|=k_{2}} \\
& \sum_{1 \leq s_{2} \leq q_{2}+k_{2}+1} \sum_{J_{2}^{1} \subset J_{1}^{1} \times \hat{J}_{2}^{2},} \sum_{J_{2}^{2} \subset J_{1}^{2} \times \hat{J}_{2}^{2},} \sum_{\left|J_{2}^{1}\right|+\left|J_{2}^{2}\right|=s_{2}} \ldots \\
& \cdots \sum_{1 \leq k_{i-1} \leq p_{i-1}+1} \sum_{\hat{J}_{i-1}^{i-1} \subset\left\{1, \ldots, p_{i-1}+1\right\},} \sum_{\left|\hat{J}_{i-1}^{i-1}\right|=k_{i-1}} \sum_{1 \leq s_{i-1} \leq q_{i-1}+k_{i-1}+1} \\
& \sum_{J_{i-1}^{1} \subset J_{i-2}^{1} \times \hat{J}_{i-1}^{i-1}, \ldots, J_{i-1}^{i-1} \subset J_{i-2}^{i-1} \times \hat{J}_{i-1}^{i-1},\left|J_{i-1}^{1}\right|+\ldots+\left|J_{i-1}^{i-1}\right|=s_{i-1}} \sum_{p_{i}+q_{i}=t_{i-1}-q_{i-1}-k_{i-1}+s_{i-1}-1} \\
& \mathrm{~b}_{q_{i}}\left(\prod_{i}^{i}\left(\bigsqcup_{i-1, J_{i-1}^{1}}^{1, i} X_{i} \cup \bigcup_{2 \leq r \leq i-1} \bigsqcup_{i-1, J_{i-1}^{r}}^{r, i} B_{i}^{r} \cup B_{i}^{i}\right)\right) .
\end{aligned}
$$

Proof. Induction on $i$. Suppose (7.1) is true. Due to Lemma 7 (De Morgan law) and Lemma 4 (Alexander's duality),

$$
\begin{gathered}
\mathrm{b}_{q_{i}}\left(\prod_{i}^{i}\left(\bigsqcup_{i-1, J_{i-1}^{1}}^{1, i} X_{i} \cup \bigcup_{2 \leq r \leq i-1} \bigsqcup_{i-1, J_{i-1}^{r}}^{r, i} B_{i}^{r} \cup B_{i}^{i}\right)\right)= \\
=\mathrm{b}_{q_{i}}\left(\left(\bigsqcup_{i}^{i}\left(\prod_{i-1, J_{i-1}^{1}}^{1, i} \widetilde{X_{i}} \cap \bigcap_{2 \leq r \leq i-1} \prod_{i-1, J_{i-1}^{r}}^{r, i} \widetilde{B_{i}^{r}} \widetilde{B_{i}^{i}}\right)\right) \tilde{)} \leq\right.
\end{gathered}
$$




$$
\begin{gathered}
\leq \mathrm{b}_{t_{i}-q_{i}-1}\left(\bigsqcup_{i}^{i}\left(\prod_{i-1, J_{i-1}^{1}}^{1, i} \widetilde{X_{i}} \cap \bigcap_{2 \leq r \leq i-1} \prod_{i-1, J_{i-1}^{r}}^{r, i} \widetilde{B_{i}^{r}} \cap \widetilde{B_{i}^{i}}\right) \cup\right. \\
\left.\cup \partial\left(I_{i}^{n_{0}+\left(p_{1}+1\right) n_{1}} \times \ldots \times I_{1}^{\left(p_{i}+1\right) n_{i}}\right)\right) .
\end{gathered}
$$

By Lemma 5 (Mayer-Vietoris inequality) the last expression does not exceed

$$
\begin{aligned}
& \sum_{1 \leq k_{i} \leq p_{i}+1} \sum_{\hat{J}_{i}^{i} \subset\left\{1, \ldots, p_{i}+1\right\},\left|\hat{J}_{i}^{i}\right|=k_{i}} \\
& \mathrm{~b}_{t_{i}-q_{i}-k_{i}}\left(\prod_{i, \hat{J}_{i}^{i}}^{i}\left(\prod_{i-1, J_{i-1}^{1}}^{1, i} \widetilde{X_{i}} \cap \bigcap_{2 \leq r \leq i-1} \prod_{i-1, J_{i-1}^{r}}^{r, i} \widetilde{B_{i}^{r}} \cap \widetilde{B_{i}^{i}}\right) \cup\right. \\
& \left.\cup \partial\left(I_{i}^{n_{0}+\left(p_{1}+1\right) n_{1}} \times \ldots \times I_{1}^{\left(p_{i}+1\right) n_{i}}\right)\right),
\end{aligned}
$$

where, by Lemma 6 ,

$$
\begin{gathered}
\mathrm{b}_{t_{i}-q_{i}-k_{i}}\left(\prod_{i, \hat{J}_{i}^{i}}^{i}\left(\prod_{i-1, J_{i-1}^{1}}^{1, i} \widetilde{X_{i}} \cap \bigcap_{2 \leq r \leq i-1} \prod_{i-1, J_{i-1}^{r}}^{r, i} \widetilde{B_{i}^{r}} \cap \widetilde{B_{i}^{i}}\right) \cup\right. \\
\left.\cup \partial\left(I_{i}^{n_{0}+\left(p_{1}+1\right) n_{1}} \times \ldots \times I_{1}^{\left(p_{i}+1\right) n_{i}}\right)\right)= \\
=\mathrm{b}_{t_{i}-q_{i}-k_{i}}\left(\prod_{i, J_{i-1}^{1} \times \hat{J}_{i}^{i}}^{1} \widetilde{X_{i}} \cap \bigcap_{2 \leq r \leq i} \prod_{i, J_{i-1}^{r} \times \hat{J}_{i}^{i}}^{r} \widetilde{B_{i}^{r}} \cup\right. \\
\left.\cup \partial\left(I_{i}^{n_{0}+\left(p_{1}+1\right) n_{1}} \times \ldots \times I_{1}^{\left(p_{i}+1\right) n_{i}}\right)\right)
\end{gathered}
$$

where $J_{i-1}^{i}=\{1\}$. By Lemma 5 (Mayer-Vietoris inequality) the last expression does not exceed

$$
\begin{aligned}
& \sum_{1 \leq s_{i} \leq q_{i}+k_{i}+1} \sum_{J_{i}^{1} \subset J_{i-1}^{1} \times \hat{J}_{i}^{i}, \ldots, J_{i}^{i} \subset J_{i-1}^{i} \times \hat{J}_{i}^{i},\left|J_{i}^{1}\right|+\ldots+\left|J_{i}^{i}\right|=s_{i}} \\
& \mathrm{~b}_{t_{i}-q_{i}-k_{i}+s_{i}-1}\left(\bigsqcup_{i, J_{i}^{1}}^{1} \widetilde{X_{i}} \cup \bigcup_{2 \leq r \leq i} \bigsqcup_{i, J_{i}^{r}}^{r} \widetilde{B_{i}^{r}} \cup \partial\left(I_{i}^{n_{0}+\left(p_{1}+1\right) n_{1}} \times \ldots \times I_{1}^{\left(p_{i}+1\right) n_{i}}\right)\right) .
\end{aligned}
$$

We have

$$
\begin{gathered}
\mathrm{b}_{t_{i}-q_{i}-k_{i}+s_{i}-1}\left(\bigsqcup_{i, J_{i}^{1}}^{1} \widetilde{X_{i}} \cup \bigcup_{2 \leq r \leq i} \bigsqcup_{i, J_{i}^{r}}^{r} \widetilde{B_{i}^{r}} \cup \partial\left(I_{i}^{n_{0}+\left(p_{1}+1\right) n_{1}} \times \ldots \times I_{1}^{\left(p_{i}+1\right) n_{i}}\right)\right)= \\
=\mathrm{b}_{t_{i}-q_{i}-k_{i}+s_{i}-1}\left(\bigsqcup_{i, J_{i}^{1}}^{1} \pi_{i+1}\left(X_{i+1}\right) \cup \bigcup_{2 \leq r \leq i} \bigsqcup_{i, J_{i}^{r}}^{r} \pi_{i+1, r-1}\left(B_{i+1}^{r}\right) \cup\right. \\
\left.\cup \pi_{i+1, i}\left(\partial\left(I_{i}^{n_{0}+\left(p_{1}+1\right) n_{1}} \times \ldots \times I_{1}^{\left(p_{i}+1\right) n_{i}}\right) \times I_{1}^{n_{i+1}}\right)\right)= \\
=\mathrm{b}_{t_{i}-q_{i}-k_{i}+s_{i}-1}\left(\pi_{i+1, i}\left(\bigsqcup_{i, J_{i}^{1}}^{1, i+1} X_{i+1} \cup \bigcup_{2 \leq r \leq i}^{\bigcup} \bigsqcup_{i, J_{i}^{r}}^{r, i+1} B_{i+1}^{r} \cup B_{i+1}^{i+1}\right)\right) .
\end{gathered}
$$

Due to Theorem 1 the last expression does not exceed

$$
\sum_{p_{i+1}+q_{i+1}=t_{i}-q_{i}-k_{i}+s_{i}-1}
$$




$$
\mathrm{b}_{q_{i+1}}\left(\prod_{i+1}^{i+1}\left(\bigsqcup_{i, J_{i}^{1}}^{1, i+1} X_{i+1} \cup \bigcup_{2 \leq r \leq i} \bigsqcup_{i, J_{i}^{r}}^{r, i+1} B_{i+1}^{r} \cup B_{i+1}^{i+1}\right)\right) \text {. }
$$

\section{Upper bounds for sub-Pfaffian sets}

We first estimate from above the number of additive terms in (7.1). These terms can be partitioned into $i-1$ groups of the kind

$$
\begin{aligned}
& \sum_{1 \leq k_{j} \leq p_{j}+1} \sum_{\hat{J}_{j}^{j} \subset\left\{1, \ldots, p_{j}+1\right\},\left|\hat{J}_{j}^{j}\right|=k_{j}} \\
& \sum_{1 \leq s_{j} \leq q_{j}+k_{j}+1} \sum_{J_{j}^{1} \subset J_{j-1}^{1} \times \hat{J}_{j}^{j}, \ldots, J_{j}^{j} \subset J_{j-1}^{j} \times \hat{J}_{j}^{j},\left|J_{j}^{1}\right|+\ldots+\left|J_{j}^{j}\right|=s_{j}} \\
& \sum_{p_{j+1}+q_{j+1}=t_{j}-q_{j}-k_{j}+s_{j}-1}
\end{aligned}
$$

where $1 \leq j \leq i-1$.

The number of terms in

$$
\sum_{1 \leq k_{j} \leq p_{j}+1} \sum_{\hat{J}_{j}^{j} \subset\left\{1, \ldots, p_{j}+1\right\},\left|\hat{J}_{j}^{j}\right|=k_{j}}
$$

is $2^{p_{j}+1}$. The number of terms in

$$
\sum_{1 \leq s_{j} \leq q_{j}+k_{j}+1} \sum_{J_{j}^{1} \subset J_{j-1}^{1} \times \hat{J}_{j}^{j}, \ldots, J_{j}^{j} \subset J_{j-1}^{j} \times \hat{J}_{j}^{j},\left|J_{j}^{1}\right|+\ldots+\left|J_{j}^{j}\right|=s_{j}}
$$

does not exceed $2^{j\left(q_{j}+k_{j}+1\right)}$. The number of terms in

$$
\sum_{p_{j+1}+q_{j+1}=t_{j}-q_{j}-k_{j}+s_{j}-1}
$$

does not exceed $t_{j}+1$.

It follows that the total number of terms in $j$ th group does not exceed

$$
2^{p_{j}+1+j\left(q_{j}+k_{j}+1\right)}\left(t_{j}+1\right) \leq 2^{O\left(j t_{j-1}\right)} .
$$

Since $t_{j}=n_{0}+n_{1}\left(p_{1}+1\right)+\ldots+n_{j}\left(p_{j}+1\right), p_{l} \leq t_{l-1}$, and therefore $t_{j} \leq 2^{j} n_{0} n_{1} \ldots n_{j}$, the number of terms in $j$ th group does not exceed $2^{O\left(j 2^{j} n_{0} n_{1} \ldots n_{j-1}\right)}$. It follows that the total number of terms in (7.1) does not exceed $2^{O\left(i^{2} 2^{i} n_{0} n_{1} \ldots n_{i-2}\right)}$.

We now find an upper bound for

$$
\mathrm{b}_{q_{\nu}}\left(\prod_{\nu}^{\nu}\left(\bigsqcup_{\nu-1, J_{\nu-1}^{1}}^{1, \nu} X_{\nu} \cup \bigcup_{2 \leq r \leq \nu-1} \bigsqcup_{\nu-1, J_{\nu-1}^{r}}^{r, \nu} B_{\nu}^{r} \cup B_{\nu}^{\nu}\right)\right) .
$$

Assume that $X_{\nu}=\left\{F\left(\mathbf{x}_{0}, \mathbf{x}_{1}, \ldots, \mathbf{x}_{\nu}\right)\right\}$, where $F$ is a quantifier-free Boolean formula with no negations having $s$ atoms of the kind $f>0, f$ 's are polynomials or Pfaffian functions of degrees less than $d$ or $(\alpha, \beta)$ respectively. In Pfaffian case, let functions $f$ be defined in an open domain $G$ by the same Pfaffian chain of order $\rho$. We assume without loss of generality that $I_{\nu}^{n_{0}+\ldots+n_{\nu}} \subset G$.

The set $\bigsqcup_{\nu-1, J_{\nu-1}^{1}}^{1, \nu} X_{\nu} \subset \mathbb{R}^{t_{\nu-1}+n_{\nu}}$ is defined by a Boolean formula with no negations having $\left|J_{\nu-1}^{1}\right| s \leq s_{\nu-1} s \leq\left(2 t_{\nu-2}+1\right) s$ atoms of degrees less than $d$ (for 
polynomials) or less than $(\alpha, \beta)$ (for Pfaffian functions) and at most $2 t_{\nu-1}+2 n_{\nu}$ linear atoms (defining $I_{1}^{t_{\nu-1}+n_{\nu}}$ ).

For any $2 \leq r \leq \nu$ the set $B_{r}^{r} \subset \mathbb{R}^{t_{r-1}+n_{r}}$ is defined by a Boolean formula with no negations having $4 t_{r-1}+2 n_{r}$ linear atomic inequalities. Therefore, all sets of the kind $B_{j}^{r}$ for $j \geq r$ are defined by Boolean formulae with no negations having $4 t_{r-1}+2\left(n_{r}+\ldots+n_{j}\right)$ linear inequalities. In particular, the set $B_{\nu}^{r} \subset \mathbb{R}^{t_{r-1}+n_{r}+\ldots+n_{\nu}}$ is defined by $4 t_{r-1}+2\left(n_{r}+\ldots+n_{\nu}\right)$ linear atomic inequalities.

For any $2 \leq r \leq \nu-1$ the set $\bigsqcup_{\nu-1, J_{\nu-1}^{r}}^{r, \nu} B_{\nu}^{r} \subset \mathbb{R}^{t_{\nu-1}+n_{\nu}}$ is defined by a Boolean formula with no negations having at most

$$
\begin{aligned}
& \left(4 t_{r-1}+2\left(n_{r}+\ldots+n_{\nu}\right)\right)\left|J_{\nu-1}^{r}\right|+2 t_{\nu-1}+2 n_{\nu} \leq \\
\leq & \left(4 t_{r-1}+2\left(n_{r}+\ldots+n_{\nu}\right)\right) s_{\nu-1}+2 t_{\nu-1}+2 n_{\nu} \leq \\
\leq & \left(4 t_{r-1}+2\left(n_{r}+\ldots+n_{\nu}\right)\right)\left(2 t_{\nu-2}+1\right)+2 t_{\nu-1}+2 n_{\nu}
\end{aligned}
$$

linear atoms.

It follows that the set $\bigcup_{2 \leq r \leq \nu-1} \bigsqcup_{\nu-1, J_{\nu-1}^{r}}^{r, \nu} B_{\nu}^{r} \subset \mathbb{R}^{t_{\nu-1}+n_{\nu}}$ is defined by a Boolean formula with no negations having at most

$$
\left(\left(4 t_{\nu-1}+2\left(n_{2}+\ldots+n_{\nu}\right)\right)\left(2 t_{\nu-2}+1\right)+2 t_{\nu-1}+2 n_{\nu}\right)(\nu-2)
$$

linear atoms.

The set

$$
\prod{ }_{\nu}^{\nu}\left(\bigsqcup_{\nu-1, J_{\nu-1}^{1}}^{1, \nu} X_{\nu} \cup \bigcup_{2 \leq r \leq \nu-1} \bigsqcup_{\nu-1, J_{\nu-1}^{r}}^{r, \nu} B_{\nu}^{r} \cup B_{\nu}^{\nu}\right) \subset \mathbb{R}^{t_{\nu}}
$$

is defined by a Boolean formula with no negations having at most

$$
\begin{gathered}
\left(\left(2 t_{\nu-2}+1\right) s+2 t_{\nu-1}+2 n_{\nu}+\left(\left(4 t_{\nu-1}+2\left(n_{2}+\ldots+n_{\nu}\right)\right)\left(2 t_{\nu-2}+1\right)+2 t_{\nu-1}+2 n_{\nu}\right)(\nu-2)\right) \cdot \\
\cdot\left(t_{\nu-1}+1\right) \leq s t_{\nu-1}^{O(1)}
\end{gathered}
$$

atoms of degrees less than $d$ for polynomials or less than $(\alpha, \beta)$ for Pfaffian functions.

Similar calculation shows that, in the Pfaffian case, the set (8.1) is defined by Pfaffian functions having the order at most $\rho\left(2 t_{\nu-2}+1\right)\left(t_{\nu-1}+1\right) \leq O\left(\rho t_{\nu-2} t_{\nu-1}\right)$.

\subsection{Polynomial case}

Let functions $f$ in formula $F$ be polynomials of degrees $\operatorname{deg}(f)<d$. Then, according to (3.1),

$$
\begin{gathered}
\mathrm{b}_{q_{\nu}}\left(\prod_{\nu}^{\nu}\left(\bigsqcup_{\nu-1, J_{\nu-1}^{1}}^{1, \nu} X_{\nu} \cup \bigcup_{2 \leq r \leq \nu-1} \bigsqcup_{\nu-1, J_{\nu-1}^{r}}^{r, \nu} B_{\nu}^{r} \cup B_{\nu}^{\nu}\right)\right) \leq \\
\leq O(d s)^{t_{\nu}} t_{\nu-1}^{O\left(t_{\nu}\right)} \leq\left(2^{\nu} d s n_{0} n_{1} \ldots n_{\nu-1}\right)^{O\left(2^{\nu} n_{0} n_{1} \ldots n_{\nu}\right)} .
\end{gathered}
$$

Using (7.1) in case $i=\nu$, we get

$$
\mathrm{b}_{q_{0}}(X) \leq\left(2^{\nu^{2}} d s n_{0} n_{1} \ldots n_{\nu-1}\right)^{O\left(2^{\nu} n_{0} n_{1} \ldots n_{\nu}\right)}
$$

(compare with (3.4) and (3.5)). 


\subsection{Pfaffian case}

Let $f$ be Pfaffian functions in an open domain $G \supset I_{1}^{n}$ of order $\rho$, degree $(\alpha, \beta)$, having a common Pfaffian chain. Then, according to (3.2),

$$
\begin{gathered}
\mathrm{b}_{q_{\nu}}\left(\prod{ }_{\nu}^{\nu}\left(\bigsqcup_{\nu-1, J_{\nu-1}^{1}}^{1, \nu} X_{\nu} \cup \bigcup_{2 \leq r \leq \nu-1} \bigsqcup_{\nu-1, J_{\nu-1}^{r}}^{r, \nu} B_{\nu}^{r} \cup B_{\nu}^{\nu}\right)\right) \leq \\
\leq 2^{O\left(\rho^{2} t_{\nu-2}^{2} t_{\nu-1}^{2}\right)}\left(s t_{\nu-1}\right)^{O\left(t_{\nu}\right)} O\left(t_{\nu} \beta+\min \left\{t_{\nu}, \rho\right\} \alpha\right)^{O\left(t_{\nu}+\rho t_{\nu-2} t_{\nu-1}\right)} \leq \\
\leq 2^{O\left(\rho^{2} 2^{4 \nu} n_{0}^{4} n_{1}^{4} \ldots n_{\nu-2}^{4} n_{\nu-1}^{2}\right)} s^{O\left(2^{\nu} n_{0} n_{1} \ldots n_{\nu}\right) .} \\
\cdot\left(2^{\nu} n_{0} n_{1} \ldots n_{\nu}(\alpha+\beta)\right)^{O\left(2^{\nu} n_{0} n_{1} \ldots n_{\nu}+\rho 2^{2 \nu} n_{0}^{2} n_{1}^{2} \ldots n_{\nu-2}^{2} n_{\nu-1}\right)} .
\end{gathered}
$$

Using (7.1) in case $i=\nu$, we get

$$
\begin{gathered}
\mathrm{b}_{q_{0}}(X) \leq 2^{O\left(\nu 2^{\nu} n_{0} n_{1} \ldots n_{\nu}+\rho^{2} 2^{4 \nu} n_{0}^{4} n_{1}^{4} \ldots n_{\nu-2}^{4} n_{\nu-1}^{2}\right)} s^{O\left(2^{\nu} n_{0} n_{1} \ldots n_{\nu}\right) .} \\
\cdot\left(n_{0} n_{1} \ldots n_{\nu}(\alpha+\beta)\right)^{O\left(2^{\nu} n_{0} n_{1} \ldots n_{\nu}+\rho 2^{2 \nu} n_{0}^{2} n_{1}^{2} \ldots n_{\nu-2}^{2} n_{\nu-1}\right)} .
\end{gathered}
$$

Introducing the notations:

$$
u_{\nu}:=2^{\nu} n_{0} n_{1} \ldots n_{\nu}, \quad v_{\nu}:=2^{2 \nu} n_{0}^{2} n_{1}^{2} \ldots n_{\nu-2}^{2} n_{\nu-1},
$$

we can rewrite this bound in a more compact form

$$
\mathrm{b}_{q_{0}}(X) \leq 2^{O\left(\nu u_{\nu}+\rho^{2} v_{\nu}^{2}\right)} s^{O\left(u_{\nu}\right)}\left(u_{\nu}(\alpha+\beta)\right)^{O\left(u_{\nu}+\rho v_{\nu}\right)} .
$$

Acknowledgements. Authors are very grateful to D. Dugger, D.B. Fuchs, and V.A. Vassiliev for useful discussions.

\section{References}

1. S. BASU, 'On bounding Betti numbers and computing Euler characteristic of semi-algebraic sets', Discrete and Comput. Geom. 22 (1999) 1-18.

2. S. BASU, R. Pollack and M.-F. Roy, 'On the combinatorial and algebraic complexity of quantifier elimination', Journal of the ACM 43 (1996) 1002-1045.

3. G.E. Collins, 'Quantifier elimination for real closed fields by cylindrical algebraic decomposition', Lecture Notes in Computer Science 33 (1975) 134-183.

4. D. Dugger, D. C. Isaksen, 'Hypercovers in topology', Preprint, 2001.

5. A. Gabrielov, N. Vorobjov, 'Complexity of cylindrical decompositions of sub-Pfaffian sets', J. Pure and Appl. Algebra 164 (2001) 179-197.

6. V. V. Goryunov, 'Semi-simplicial resolutions and homology of images and discriminants of mappings', Proc. London Math. Soc. 70 (1995) 363-385.

7. V. V. Goryunov, D. M. Q. Mond, 'Vanishing cohomology of singularities of mappings', Compositio Math. 89 (1993) 45-80.

8. J. Heintz, M.-F. Roy and P. Solernó, 'Sur la complexité du principe de Tarski-Seidenberg', Bull. Soc. Math. France 118 (1990) 101-126.

9. K. Houston, 'An introduction to the image computing spectral sequence', in: Singularity Theory 305-324, London Math. Soc. Lecture Notes Ser., 263, Combridge Univ. Press, Cambridge, 1999.

10. A. G. Khovanskil, Fewnomials, Translations of Mathematical Monographs 88 (AMS, Providence, RI, 1991).

11. W. S. Massey, A Basic Course in Algebraic Topology (Springer-Verlag, New York, 1991).

12. J. Milnor, 'On the Betti numbers of real varieties', Proc. AMS 15 (1964) 275-280.

13. J. L. Montaña, J. E. Morais and L. M. Pardo, 'Lower bounds for arithmetic networks II: sum of Betti numbers', Applic. Algebra Eng. Commun. Comp. 7 (1996) 41-51.

14. O. A. Oleinik, 'Estimates of the Betti numbers of real algebraic hypersurfaces' (Russian), Mat. Sbornik 28 (1951) 635-640. 
15. O. A. Oleinik, I. G. Petrovskir, 'On the topology of real algebraic hypersurfaces' (Russian), Izv. Acad. Nauk SSSR 13 (1949) 389-402. English transl.: Amer. Math. Soc. Transl. 7 (1962) 399-417.

16. J. RENEGAR, 'On the computetional complexity and geometry of the first order theory of reals, I-III', J. Symbolic Comput. 13 (1992) 255-352.

17. E. Spanier, Algebraic Topology (Springer-Verlag, New York-Berlin, 1981).

18. R. Тном, 'Sur l'homologie des variétés algebriques réelles', in: Differential and Combinatorial Topology 255-265 (Princeton University Press, Princeton, 1965).

19. V. A. VASSILIEv, Complements of Discriminants of Smooth Maps: Topology and Applications, Translations of Mathematical Monographs, 98 (AMS, Providence, RI, 1992).

20. H. R. Wüthrich, 'Ein Entschedungsvefahren für die Theorie der reell-abgeschlossenen Körper', Lecture Notes in Computer Science 43 (1976) 138-162.

21. A. C. C. YAO, 'Decision tree complexity and Betti numbers', in: Proceedings of 26th ACM Symp. on Theory of Computing, Montreal, Canada 615-624 (ACM Press, New York, 1994).

22. T. Zell, 'Betti numbers of semi-Pfaffian sets', J. Pure Appl. Algebra 139 (1999) 323-338.

A. Gabrielov and T. Zell

Department of Mathematics, Purdue University, West Lafayette, IN 47907

$U S A$

agabriel@math.purdue.edu tzell@math.purdue.edu

\section{N. Vorobjov}

Department of Computer Science, University of Bath, Bath BA2 7AY

nnv@cs.bath.ac.uk 